\title{
ON THE SCHWARZ REFLECTION PRINCIPLE
}

\author{
BY
}

\section{J. S. HWANG}

\begin{abstract}
Recently, we have solved a long outstanding problem of A. J. Lohwater (1953) by showing that if $f(z)$ is meromorphic in $|z|<1$ whose radial limits have modulus 1 for almost all points on an $\operatorname{arc} A$ of $|z|=1$, and if $P$ is a singular point of $f(z)$ on $A$, then every value of modulus 1 which is not in the range of $f(z)$ at $P$ is an asymptotic value of $f(z)$ at some point of each subarc of $A$ containing the point $P$.

Lohwater proved this theorem for functions of bounded characteristic and he made a comment that his method is not, in general, applicable to functions of unbounded characteristic. In this paper, we shall present an alternative proof of the above theorem based on the very method of Lohwater.
\end{abstract}

1. Introduction. In [6, Theorem 3], Lohwater proved the following result: Let $f(z)$ be meromorphic in the unit disk $D=\{z:|z|<1\}$ with bounded characteristic in the sense of Nevanlinna (see [2, p. 38]) and let the radial $\operatorname{limits}_{\lim } f\left(r e^{i \theta}\right)=f\left(e^{i \theta}\right)$ have modulus 1 for almost all points $e^{i \theta} \in A=\left\{e^{i \theta}: \alpha<\theta<\beta\right\}$. If $P$ is a singular point of $f(z)$ on $A$, then every value of modulus 1 which is not in the range of $f(z)$ at $P$ is an asymptotic value of $f(z)$ at some point of each subarc of $A$ containing the point $P$. He then asked as to whether this result is still true if $f(z)$ is not of bounded characteristic (see [6, p. 156]). Recently, in [4], we have solved this problem in the affirmative sense as follows.

THEOREM 1. Let $f(z)$ be meromorphic in $D$ and let the radial limits $\lim _{r \rightarrow 1} f\left(r e^{i \theta}\right)=$ $f\left(e^{i \theta}\right)$ exist and have the modulus 1 for almost all $e^{i \theta} \in A=\left\{e^{i \theta}: \alpha<\theta<\beta\right\}$. If $P$ is a singular point of $f(z)$ on $A$, then every value of modulus 1 which is not in the range of $f(z)$ at $P$ is an asymptotic value of $f(z)$ at some point of each subarc of $A$ containing the point $P$.

The first purpose in this paper is to present a different proof of Theorem 1 based on the method of Lohwater which was apparently thought to be impossible by him [6, p. 156]. Next, we introduce three classes of functions $f(z)$ satisfying the condition $\lim _{r \rightarrow 1}\left|f\left(r e^{i \theta}\right)\right|=1$ for almost all points on $A$, but not necessary to have radial limits of modulus 1 . We then prove the analogues of Theorem 1 with some additional conditions. Finally, we study a class of functions which belong to Seidel's class $U$ (see [2, p. 107]) and we prove some extension of Seidel's theorem [7, Theorem 4].

Received by the editors November 26, 1980 and, in revised form, May 6, 1981.

1980 Mathematics Subject Classification. Primary 30C80; Secondary 30D40.

Key words and phrases. Asymptotic behaviour, bounded characteristic, reflection principle, Seidel's class $U$. 
2. Proof of Theorem 1. To prove this, we shall need the following result of Lohwater [6, Theorem 2].

LeMma 1. Let $g(z)$ be meromorphic in D such that $\lim _{r \rightarrow 1}\left|g\left(r e^{i \theta}\right)\right|=1$ for almost all $e^{i \theta} \in A=\left\{e^{i \theta}: \alpha<\theta<\beta\right\}$. If $g(z)$ has no zeros or poles in the region $0 \leqslant 1-\varepsilon$ $<|z|<1, \alpha<\arg z<\beta$, then a necessary and sufficient condition that $g(z)$ can be continued analytically beyond $A$ is that $g(z)$ admits neither 0 nor $\infty$ as asymptotic values on $A$.

With the help of Lemma 1 and the method of [6, Theorem 3], we are now able to prove Theorem 1. Let $\zeta=e^{i a}$ be a point not in the range of $f(z)$ at $P$, then the function

$$
g(z)=\exp \left(\frac{f(z)+\zeta}{f(z)-\zeta}\right)
$$

is analytic without zeros in the region $G_{\delta}(P)=\{z: z \in D$ and $|z-P|<\delta\}$ for sufficiently small $\delta>0$. To prove the assertion, we shall consider two cases: Either there is a sequence of points $e^{i \theta_{n}}$ tending to $P$ for which

$$
\lim _{r \rightarrow 1} f\left(r e^{i \theta_{n}}\right)=\zeta, \quad n=1,2, \ldots,
$$

or not. Clearly, if the first case occurs then there is nothing more to prove. We may therefore assume that whenever the radial limit exists at $e^{i \theta}$, we have

$$
\lim _{r \rightarrow 1} f\left(r e^{i \theta}\right) \neq \zeta \text {. }
$$

We shall prove that

$$
\lim _{r \rightarrow 1}\left|g\left(r e^{i \theta}\right)\right|=1, \quad \text { for almost all } \theta \in(\alpha, \beta) .
$$

For this, we write

$$
f\left(r e^{i \theta}\right)=\rho e^{i \phi} \quad \text { where } r<1, \rho=\rho(r, \theta), \text { and } \phi=\phi(r, \theta) .
$$

Let $\theta_{0} \in(\alpha, \beta)$ be an arbitrary point for which the radial $\operatorname{limit}_{r \rightarrow 1} f\left(r e^{i \theta_{0}}\right)=$ $f\left(e^{i \theta_{0}}\right)$ exists and has the modulus 1 . Then by (2) and (4) we have

$$
\lim _{r \rightarrow 1} \rho\left(r, \theta_{0}\right)=1 \text { and } \lim _{r \rightarrow 1} \phi\left(r, \theta_{0}\right)=\phi_{0} \neq a .
$$

Since the quotient

$$
\frac{f(z)+\zeta}{f(z)-\zeta}=\frac{|f(z)|^{2}-1+\zeta \overline{f(z)}-\bar{\zeta} f(z)}{|f(z)-\zeta|^{2}},
$$

it follows from (1), (2), (4) and (5) that

$$
\lim _{r \rightarrow 1}\left|g\left(r e^{i \theta_{0}}\right)\right|=\exp \left\{\lim _{r \rightarrow 1} \frac{\rho\left(r, \theta_{0}\right)^{2}-1}{\left|\rho e^{i \phi_{0}}-e^{i a}\right|^{2}}\right\}=1 .
$$

This yields (3) due to the fact that the radial limits $f\left(e^{i \theta_{0}}\right)$ exist and are of modulus 1 almost everywhere on $A$. Thus the function $g(z)$ satisfies the hypothesis of Lemma 1 . Moreover, it is easy to see that the point $P$ is also a singular point of $g(z)$. It then follows from Lemma 1 that there exists an arc $L$ lying in $D$ and terminating either at 
$P$ or at a point $P^{\prime}$ of $|z|=1$ as close to $P$ as we please, such that the function $g(z)$ tends to 0 or $\infty$ as $z \rightarrow P$ or $P^{\prime}$ along $L$. This yields that $f(z)$ admits $\zeta$ as an asymptotic value either at $P$ or $P^{\prime}$ and the proof is complete.

Note that by a theorem of W. Rudin [6, p. 155], Lohwater constructed a function $f(z)$ which has the radial limit 1 almost everywhere on $|z|=1$, and the associated function $g(z)$, defined by (1), where $\zeta=1$, has the property that $\lim _{r \rightarrow 1}\left|g\left(r e^{i \theta}\right)\right|=\infty$ for almost all $e^{i \theta}$. However, in this case, the function $f(z)$ has the asymptotic value $\zeta=1$ almost everywhere on $|z|=1$ and of course close to any singular point $P$ on $|z|=1$, so that the assertion of Theorem 1 becomes obvious if the value $\zeta=1$ is not in the range of $f(z)$ at $P$. This example is in a sense misleading to overlook the difficulty of Theorem 1 .

3. Generalized Seidel's class $U$. Following E. F. Collingwood and A. J. Lohwater [2, p. 107], we shall denote by $U$ the class of all functions $f(z)$ meromorphic in $D$ for which the radial limits $f\left(e^{i \theta}\right)$ exist and have modulus 1 for almost all points $e^{i \theta}$. Instead of a function $f(z)$, Lohwater has also considered its modulus $|f(z)|$ [6]. In this connection, we shall now define three generalized classes $|U|,|U|^{-}$, and $|U|^{+}$ as follows. A meromorphic function $f \in|U|$, if for almost all points $e^{\mathrm{i} \theta}$ the radial limit of the modulus $\lim _{r \rightarrow 1}\left|f\left(r e^{i \theta}\right)\right|=1$. In this case, the function $f(z)$ may not have radial limits almost everywhere on $|z|=1$. Clearly, the class $U$ is a subclass of $|U|$. Moreover, we call a function $f \in|U|^{-}$or $|U|^{+}$if for almost all points $e^{i \theta}$, the radial limit of the modulus $\left|f\left(r e^{i \theta}\right)\right|$ tends to 1 from below or above respectively as $r \rightarrow 1$. In this case, the class $U$ needs not be a subclass of $|U|^{-}$or $|U|^{+}$, but both $|U|^{-}$and $|U|^{+}$are subclasses of $|U|$. Notice that the above definitions can go through in a local sense. For instance, we say that $f \in|U|$ on an $\operatorname{arc} A$ of $|z|=1$, if for almost all points $e^{i \theta} \in A, \lim _{r \rightarrow 1}\left|f\left(r e^{i \theta}\right)\right|=1$. Clearly, if $f \in|U|$, then $f \in|U|$ on any arc of $|z|=1$.

We shall now construct functions belonging to each of the above three classes.

THEOREM 2. There exist three functions $f \in|U|-U \cup|U|^{-} U|U|^{+}, f^{-} \in|U|^{-}-U$ $\cup|U|^{+}$, and $f^{+} \in|U|^{+}-U \cup|U|^{-}$.

Proof. We shall first construct the function $f(z)$. For this, we let $A$ be an arc on $|z|=1$ and let $\Gamma$ be a continuous curve (not closed) surrounding and converging to $A$. Denote by $E$ a set of first category and of measure $2 \pi$ on $|z|=1$, and $\left\{R_{\theta}\right\}$ a family of radial segments terminating at the points $e^{i \theta} \in E$, where none of the closure $\bar{R}_{\theta}$ contains the origin. Let $g(z)$ be a function mapping each $R_{\theta}$ homeomorphically onto $\Gamma$, then, by a well-known theorem of Urysohn, the function $g(z)$ can be extended to be continuous through $D$. It follows from an approximation theorem of F. Bagemihl and W. Seidel or W. Rudin (see [2, Theorem 8.11]) that there is a function $f(z)$ analytic in $D$ such that, for every $e^{i \theta} \in E$,

$$
f(z)-g(z) \rightarrow 0, \quad \text { as } z \rightarrow e^{i \theta} \text { on } R_{\theta} .
$$

Since the curve $\Gamma$ converges to $A$, it follows that

$$
\lim _{r \rightarrow 1}\left|f\left(r e^{i \theta}\right)\right|=\lim _{r \rightarrow 1}\left|g\left(r e^{i \theta}\right)\right|=1 \quad \text { for each } e^{i \theta} \in E .
$$

Moreover, the set $E$ has measure $2 \pi$, so that the function $f \in|U|$. 
It remains to show that $f \notin U \cup|U|^{-} \cup|U|^{+}$. This, however, is simple, because the curve $\Gamma$ surrounds the arc $A$, so that the radial cluster set $C_{\rho}\left(f, e^{i \theta}\right)$ coincides with $A$ for every point $e^{i \theta} \in E$, and the limit $\left|f\left(r e^{i \theta}\right)\right|$ tends to 1 alternatively from below and above, as $r \rightarrow 1$.

Finally, we shall construct both functions $f^{-}(z)$ and $f^{+}(z)$. To do this, we need only require the curve $\Gamma$ tend to $A$ from the interior or exterior of $D$ respectively. Then by the same argument as before we can obtain the desired functions. This proves the assertion.

4. Boundary behaviour of class $|U|$. Before proving some extensions of Theorem 1 , we shall now study the boundary behaviour of the class $|U|$. For convenience, if $G$ is a domain we shall denote by $\partial G$ the boundary of $G$ and in particular, if $G=D$, the unit disk, we shall write the unit circle by $C=\{z:|z|=1\}$.

According to an extension of Löwner's theorem [9, Theorem VIII.30] and a theorem of Bagemihl on ambiguous points [2, Theorem 4.12], we have the following.

THEOREM 3. Let $f(z)$ be a function in the class $|U|$, such that for $n>1$ the domain $G_{n}=\{z: z \in D$ and $|f(z)|>n\}$ is not empty, and let $H_{n}$ be a component of $G_{n}$. If $z(w)$ is a conformal mapping from $D_{w}=\{w:|w|<1\}$ onto $H_{n}$ and if $E$ is the set of all points on $|w|=1$ such that for each $e^{i \theta} \in E$ the radial limit $\lim _{r \rightarrow 1} z\left(r e^{i \theta}\right)$ exists and has the modulus 1 , then the set $E$ is of measure 0.

Proof. Let $E^{*}$ be the image of $E$ on $|z|=1$, i.e. $E^{*}=\left\{e^{i \phi}=z\left(e^{i \theta}\right): e^{i \theta} \in E\right\}$. Since $E$ is a Borel set, it follows from the aforementioned theorem of Löwner that $E^{*}$ is a Borel set and the measures $|E| \leqslant\left|E^{*}\right|$. Therefore, to prove the assertion $|E|=0$, it is sufficient to show that $\left|E^{*}\right|=0$.

We now consider the function $f \in|U|$. Let $E_{1}$ be the set of all points $e^{i \phi}$ on $C$ such that $\lim _{r \rightarrow 1}\left|f\left(r e^{i \phi}\right)\right|=1$, then the measure $\left|E_{1}\right|=2 \pi$. Denote by $C_{\rho}\left(f, e^{i \phi}\right)$ the radial cluster set of $f(z)$ at the point $e^{i \phi}$, then clearly we have

$$
C_{\rho}\left(f, e^{i \phi}\right) \subset\{\zeta:|\zeta|=1\} \quad \text { for each } e^{i \phi} \in E_{1} .
$$

On the other hand, if $e^{i \phi} \in E^{*}$, then there is a point $e^{i \theta}$ on $|w|=1$ for which $\lim _{r \rightarrow 1} z\left(r e^{i \theta}\right)=e^{i \phi}$. Let $r_{\theta}$ be the radius in $D_{w}$ ending at $e^{i \theta}$ and let $\gamma_{\phi}=z\left(r_{\theta}\right)$, then $\gamma_{\phi}$ is an arc lying in $H_{n}$ and ending at $e^{i \phi}$. Denote by $C_{\gamma_{\phi}}\left(f, e^{i \phi}\right)$ the cluster set of $f(z)$ along $\gamma_{\phi}$, then we have

$$
C_{\gamma_{\phi}}\left(f, e^{i \phi}\right) \subset\{\zeta:|\zeta| \geqslant n\} \quad \text { for each } e^{i \phi} \in E^{*} .
$$

To prove $\left|E^{*}\right|=0$, we suppose on the contrary that $\left|E^{*}\right|>0$, then the measure of the intersection $\left|E^{*} \cap E_{1}\right|>0$. Since $n>1$, it follows from (6) and (7) that the following set is empty

$$
C_{\rho}\left(f, e^{i \phi}\right) \cap C_{\gamma_{\phi}}\left(f, e^{i \phi}\right)=\varnothing \quad \text { for each } e^{i \phi} \in E^{*} \cap E_{1} .
$$

This contradicts the aforementioned theorem of Bagemihl and the proof is complete.

We notice that the above Theorem 3 can be extended. For this, we let $B_{m}$ be the class of all functions $f(z)$ meromorphic in $D$ for which the radial cluster sets $C_{\rho}\left(f, e^{i \phi}\right) \subset\{\zeta:|\zeta| \leqslant m\}$ for almost all points $e^{i \phi}$. Locally, we say that $f \in B_{m}$ on an $\operatorname{arc} A$ of $|z|=1$, if the above property holds for almost all points $e^{\mathrm{i} \phi} \in A$. 
COROLlaRY 1. Theorem 3 is still true if the class $|U|$ is replaces by the class $B_{m}$ restricted on an arc $A(\alpha, \beta)=\left\{e^{i \theta}: \alpha<\theta<\beta\right\}$, where $G_{n}=\{z: \alpha<\arg z<\beta$ and $|f(z)|>n\}$, and $m<n$.

Proof. The proof can be easily finished just by replacing equation (6) by

$$
C_{\rho}\left(f, e^{i \phi}\right) \subset\{\zeta:|\zeta| \leqslant m\}, \text { where } m<n .
$$

5. Generalizations of Theorem 1. In view of Theorem 1 and the definitions of $U$ and $|U|$, we can see that Theorem 1 holds for any function $f(z)$ in the class $U$. We may therefore ask as to whether Theorem 1 is still true if the class $U$ is replaced by the class $|U|$. This question will be answered partially by the following two results. However, we believe that the answer should be affirmative.

THEOREM 4. If $f \in|U|^{-} \cup|U|^{+}$on an arc $A$ of $C$ and if $P$ is a sungular point of $f(z)$ on $A$, then every value of modulus 1 which is not in the range of $f(z)$ at $P$ is an asymptotic value of $f(z)$ at some point of each subarc of $A$ containing the point $P$.

Proof. We shall first consider a function $f \in|U|^{-}$on the arc $A$. As before, we let $\zeta=e^{i a}$ be a point not in the range of $f(z)$ at $P$ and let $g(z)$ be the function defined by (1) which is analytic and has no zeros in the region $G_{\delta}(P)$ defined in (1). Denote by $E$ the set of all points $e^{i \theta} \in A$ such that $\left|f\left(r e^{i \theta}\right)\right|$ tends to 1 from below, as $r \rightarrow 1$, then the measure $|E|=|A|$. Let $\partial G_{\delta}(P)$ be the boundary of $G_{\delta}(P)$. We shall prove that for each point $e^{i \theta} \in E \cap \partial G_{\delta}(P)$ there is a radial segment $R_{\theta}$ such that the function $g(z)$ is bounded by one on $R_{\theta}$. To do this, we write $f\left(r e^{i \theta}\right)$ as in (4), then the real part

$$
\operatorname{Re} \frac{f\left(r e^{i \theta}\right)+\zeta}{f\left(r e^{i \theta}\right)-\zeta}=\frac{\rho^{2}-1}{\left|f\left(r e^{i \theta}\right)-\zeta\right|^{2}},
$$

where $\rho=\rho(r, \theta)=\left|f\left(r e^{i \theta}\right)\right|$ and $r<1$. Since $e^{i \theta} \in E$, there is a number $r_{0}>0$ such that $r_{0} \leqslant r \leqslant 1$ implies $\left|f\left(r e^{i \theta}\right)\right| \leqslant 1$. Moreover, the point $e^{i \theta} \in \partial G_{\delta}(P)$, so that there is a number $r_{1}$ for which $f\left(r e^{i \theta}\right) \neq \zeta$ provided $r_{1} \leqslant r<1$ where $r_{1} e^{i \theta} \in G_{\delta}(P)$. Let $R_{\theta}=\left\{r e^{i \theta}: \max \left(r_{0}, r_{1}\right) \leqslant r \leqslant 1\right\}$, then by (8) we conclude that on the radial segment $R_{\theta}$

$$
\left|g\left(r e^{i \theta}\right)\right|=\exp \left\{\operatorname{Re} \frac{f\left(r e^{i \theta}\right)+\zeta}{f\left(r e^{i \theta}\right)-\zeta}\right\} \leqslant 1 \quad \text { for each } e^{i \theta} \in E \cap \partial G_{\delta}(P) .
$$

We now choose two points $e^{i \alpha}, e^{i \beta} \in E \cap \partial G_{\delta}(P)$ such that the point $P$ lies on the arc between $e^{i \alpha}$ and $e^{i \beta}$. Let $R_{\alpha}$ and $R_{\beta}$ be the associated radial segments for which (9) holds. Let $L$ be the line segment joining the endpoints of $R_{\alpha}$ and $R_{\beta}$ which lie within the domain $G_{\delta}(P)$. Then we have for some $M>1$,

$$
|g(z)| \leqslant M \text { for every } z \in R_{\alpha} \cup L \cup R_{\beta} .
$$

Denote by $G$ the region bounded by $R_{\alpha}, L, R_{\beta}$, and the $\operatorname{arc} A(\alpha, \beta)=\left\{e^{i \phi}\right.$ : $\alpha<\phi<\beta\}$. If the function $g(z)$ is bounded in $G$, then the assertion follows from Lohwater's theorem [6, Theorem 3]. Therefore we need only consider the case that $g(z)$ is unbounded in $G$. Let $n>M$ be a positive integer and let $z_{n}$ be a point in $G$ 
for which $\left|g\left(z_{n}\right)\right|>n$. Define the domain $G_{n}=\{z:|g(z)|>n\}$ and choose a component $H_{n}$ of $G_{n}$ for which $z_{n} \in H_{n}$.

We shall prove that $H_{n} \subset G$. Suppose on the contrary that $H_{n} \not \subset G$, then by (10) and the maximum principle we find that $\bar{H}_{n} \cap A(\alpha, \beta) \neq \varnothing$. In this case, we consider the function $1 / g(z)$ which is bounded by $1 / n$ on $H_{n}$ and therefore has radial limits almost everywhere on $\bar{H}_{n} \cap A(\alpha, \beta)$. It follows from (3) that the function $g(z)$ has radial limits of modulus 1 almost everywhere on $\bar{H}_{n} \cap A(\alpha, \beta)$. This clearly is impossible because $|g(z)|>n$ for any $z \in H_{n}$. We thus conclude that $H_{n} \subset G$.

The key point to find the desired asymptotic value is to prove that the function $g(z)$ is unbounded in $H_{n}$. For this, we let $H_{n}^{*}$ be the smallest simply connected domain containing $H_{n}$. Since $H_{n} \subset G$, we have $H_{n}^{*} \subset D$, and the boundaries of $H_{n}^{*}$ and $H_{n}$ on $C$ are the same. Let $z=z(w)$ be a conformal mapping from $D_{w}=\{w$ : $|w|<1\}$ onto $H_{n}^{*}$. Denote by $E_{1}$ the set of all points on $|w|=1$ such that for each $e^{i \theta} \in E_{1}$ the radial limit $\lim _{r \rightarrow 1} z\left(r e^{i \theta}\right)$ exists and has the modulus 1 . In view of (8) and (9), we can see that the radial cluster sets $C_{\rho}\left(g, e^{i \theta}\right) \subset\{\lambda:|\lambda| \leqslant 1\}$ for each $e^{i \theta} \in E$, where $|E|=|A|$, so that the function $g \in B_{1}$ on the arc $A$. Since the number $n>M>1$, the domain $H_{n}^{*} \subset D$, and the boundary of $H_{n}^{*}$ on $C$ is the same as that of $H_{n}$, it follows from Corollary 1 that the set $E_{1}$ has measure 0 . This concludes that for almost all points $e^{i \theta}$ on $|w|=1$ the radial limits $\lim _{r \rightarrow 1} z\left(r e^{i \theta}\right)$ exist and have modulus less than 1.

We now consider the function $h(w)=g(z(w))$. By what we have just proved, we find that

$$
\lim _{r \rightarrow 1} h\left(r e^{i \theta}\right)=n \quad \text { for almost all } e^{i \theta} \text { on }|w|=1 .
$$

If $h(w)$ were bounded in $D_{w}$, then by the strong form of the maximum principle [2, Theorem 5.3], the function $h(w)$ would be bounded by $n$ through $D_{w}$. This in turn implies that the function $g(z)$ is bounded by $n$ in the component $H_{n}$ of $G_{n}$, contradicting the definition of $G_{n}=\{z:|g(z)|>n\}$. We thus conclude that $g(z)$ is unbounded in $H_{n}$.

Finally, by the same argument as in [4], we can construct a path $\Gamma$ ending at a point $Q \in A(\alpha, \beta)$ for which the function $g(z)$ tends to $\infty$ along $\Gamma$. This concludes that the function $f(z)$ tends to the value $\zeta$ along $\Gamma$ and proves the assertion for the case $f \in|U|^{-}$.

On the other hand, if $f \in|U|^{+}$, then instead of the associated function $g(z)$, we consider its inverse, i.e.

$$
g(z)^{-1}=\exp \left(-\frac{f(z)+\zeta}{f(z)-\zeta}\right)
$$

By the same argument, we can obtain the assertion. This completes the proof.

Unfortunately, we have not been able to prove Theorem 4 for any function $f \in|U|$. However, if the function $f(z)$ is required to be analytic in $D$ then the assertion of Theorem 4 is still true. More general, we state and prove the following. 
THEOREM 5. If $f \in|U|$ on an arc $A$ of $|z|=1$, and if $P$ is a singular point of $f(z)$ on $A$ such that the function $f(z)$ is analytic in a relative vicinity of $P$, then every value of modulus 1 which is not in the range of $f(z)$ at $P$ is an asymptotic value of $f(z)$ at some point of each subarc of $A$ containing the point $P$.

Proof. As before, let $\zeta=e^{i a}$ be a point not in the range of $f(z)$ at $P, f(z)$ analytic in the region $G_{\delta}(P)$, and $E=\left\{e^{i \theta}: e^{i \theta} \in A\right.$ and $\left.\lim _{r \rightarrow 1}\left|f\left(r e^{i \theta}\right)\right|=1\right\}$. Choose two sequences $\left\{e^{i \alpha_{n}}\right\}$ and $\left\{e^{i \beta_{n}}\right\}$ of points in $E$ such that $e^{i \alpha_{n}} \uparrow P$ and $e^{i \beta_{n}} \downarrow P$ as $n \rightarrow \infty$, where $\alpha_{n}<\beta_{n}$. For each $e^{i \theta}$, we let $R(\theta)$ be the radius ending at $e^{i \theta}$. Let $G_{n}^{\alpha}$ be the subregion of $G_{\delta}(P)$ bounded by the radii $R\left(\alpha_{n}\right)$ and $R\left(\alpha_{n+1}\right)$, and the boundary of $G_{\delta}(P)$, and let $G_{n}^{\beta}$ be the subregion of $G_{\delta}(P)$ bounded by the radii $R\left(\beta_{n}\right)$ and $R\left(\beta_{n+1}\right)$, and the boundary of $G_{\delta}(P)$. We shall consider three cases about the boundedness of the function $f(z)$, namely, either bounded in each $G_{n}^{\alpha}$, or $G_{n}^{\beta}$, or both of them. Notice that in each case the function $f(z)$ is not necessary to be uniformly bounded through all $n$. However, the condition of boundedness guarantees that the function $f(z)$ have radial limits almost everywhere on the boundary of each $G_{n}^{\alpha}$ or $G_{n}^{\beta}$. Therefore, if the last case occurs then $f(z)$ has radial limits almost everywhere on a subarc $B$ of $A$ which contains the point $P$. In this case, the assertion follows from [4]. Turning to the first case, we may assume that there is a subsequence $\left\{n_{k}\right\}$ such that $f(z)$ is unbounded in each $G_{n_{k}}^{\beta}$, otherwise we would reduce to the last case and we are done. Let $\gamma_{n_{k}}$ be the portion of $\partial G_{n_{k}}^{\beta}$ which lies inside $D$. Since $f(z)$ is analytic in $G_{\delta}(P)$, we may, without loss of generality, assume that $f(z)$ has no poles on the portion of $\partial G_{\delta}(P)$ which lies inside $D$. This together with the choices $e^{i \beta_{n}} \in E$ yields that

$$
|f(z)| \leqslant M_{n_{k}}<\infty, \quad \text { for each } z \in \gamma_{n_{k}} .
$$

Since $f(z)$ is unbounded in the region $G_{n_{k}}^{\beta}$, by (11) and the same argument as before we conclude that $f(z)$ has the asymptotic value $\infty$ along a path $\Gamma_{n_{k}}$ lying in $G_{n_{k}}^{\beta}$ and tending to a point $P_{n_{k}}$ on $C$. Since $e^{i \beta_{n}} \downarrow P$, it follows that $P_{n_{k}} \downarrow P$, as $k \rightarrow \infty$.

We now consider the function

$$
g(z)=1 /(f(z)-\zeta), \text { where } f(z) \neq \zeta \text { in } G_{\delta}(P) .
$$

By what we have just proved, the function $g(z)$ has the asymptotic value 0 along each $\Gamma_{n_{k}}$. Since we consider the function $f(z)$ to be bounded in each subregion $G_{n}^{\alpha}$, the function $g(z)$ has finite radial limits almost everywhere on each arc of the form $\left\{e^{i \theta}: \alpha_{n}<\theta<\arg P\right\}$. For each $k$ (associating with the subsequence $n_{k}$ ), we let $R_{k}$ be a radial segment lying in $G_{k}^{\alpha}$ such that $g(z)$ has a finite radial limit along $R_{k}$, and let $L_{k}$ be the line segment joining the endpoints of $\Gamma_{n_{k}}$ and $R_{k}$ which lie within $G_{\delta}(P)$. Then we have

$$
|g(z)| \leqslant M_{k}<\infty, \quad \text { for each } z \in \Gamma_{n_{k}} \cup L_{k} \cup R_{k} .
$$

Denote by $H_{k}$ the region bounded by $\Gamma_{n_{k}}, L_{k}, R_{k}$, and the arc $B_{k}$ on $|z|=1$ connecting the endpoints of $\Gamma_{n_{k}}$ and $R_{k}$. If the funciton $g(z)$ is bounded in $H_{k}$, then the function $f(z)$ has radial limits almost everywhere on $B_{k}$. This reduces to the last case and we are done. We may, therefore, assume that $g(z)$ is unbounded in $H_{k}$. It then follows from (12) that there is a path $\gamma \subset H_{k}$ ending at a point on $B_{k}$ such that 
$g(z)$ has the asymptotic value $\infty$ along $\gamma$. This in turn implies that the function $f(z)$ has the asymptotic value $\zeta$ along $\gamma$ and proves the assertion for the first case. The second case can be proved by the same argument. This completes the proof.

Notice that the above proof actually yields the following more general result and we omit the proof.

COROLlaRY 2. If $f \in|U|$ on an arc $A$ of $C$ and if $f(z)$ has radial limits on a sequence $\left\{e^{i \alpha_{n}}\right\}$ which tends to the singular point $P$ from both sides, then the assertion of Theorem 5 is still true.

6. Omitted values. In view of Theorem 1 , we may ask whether there exists a function $f \in U$ which omits a prescribed value $\zeta$ of modulus 1 in $D$. This question will be answered by the following.

THEOREM 6. Let $\zeta=e^{i a} \neq 1$, then there is a function $f \in U$ which omits the value $\zeta$ in $D$.

Proof. In view of [6, p. 155], there is a function $g(z)$, analytic in $D$ and having the radial limit 1 almost everywhere on $|z|=1$. We set

$$
f(z)=\frac{\zeta[(1+\zeta) g(z)+1-\zeta]}{(1+\zeta) g(z)-1+\zeta}, \quad \text { where } \zeta \neq 1 .
$$

Clearly, we have for each $e^{i \theta}$,

$$
\lim _{r \rightarrow 1} g\left(r e^{i \theta}\right)=1 \text { implies } \lim _{r \rightarrow 1} f\left(r e^{i \theta}\right)=1,
$$

so that the function $f \in U$.

It follows from (13) that $f(z)=\zeta$ if and only if $g(z)=\infty$. Since $g(z)$ is analytic in $D$, the function $f(z)$ must omit the value $\zeta$ in $D$. This proves the theorem.

Notice that Theorem 6 is still true if the class $U$ is replaced by the class $|U|$. Also notice that as far as the omitted values are concerned, Theorem 1 can be extended to the class $|U|$.

THEOREM 7. If $f \in|U|$ on an arc $A$ of $|z|=1$, and if $f$ omits three values in a relative vicinity $V$ of $P$, then the assertion of Theorem 1 is still true.

Proof. According to a well-known theorem of Montel (see O. Lehto and K. I. Virtanen [5, p. 53]), the function $f(z)$ is normal in $V$. It follows from a result of $\mathrm{F}$. Bagemihl and W. Seidel [1, Corollary 1] that $f(z)$ has angular limits on a dense subset of $A$. The assertion now is a consequence of Corollary 2.

7. The range of $U_{1}$-class. In this section, we shall improve a result of Seidel [8, p. 211]. For this, we let $U_{1}$ be the class of all functions $f(z)$, analytic in $D$, bounded by 1 , having radial limits $\left|f\left(e^{i \theta}\right)\right|=1$ almost everywhere on $|z|=1$, containing an isolated singularity at $e^{i \alpha}$, and omitting a value $v$ with $|v|<1$ in a relative vicinity of $e^{i \alpha}$. Functions of this kind do exist, for instance, the typical function $f_{1}(z)=$ $e^{(z+1) /(z-1)} \in U_{1}$. Based on an application of Seidel's theorem [7, Theorem 4], we proved that if $f \in U_{1}$, then the range of $f(z)$ covers the interior of some circle of radius $\frac{1}{2}[3$, Theorem 7$]$, where the condition of omitting value is not required. We shall now improve this result in the following two sides sense. 
THEOREM 8. Let $f \in U_{1}$ and let $f(z)$ have an isolated singularity at a point $e^{i \alpha}$ and omit a value $v$ with $|v|<1$ in a relative vicinity of $e^{i \alpha}$. If $D^{-}$and $D^{+}$denote respectively the lower and upper half-disk in $D$ separated by the diameter passing through the point $e^{i \alpha}$, then both of the ranges $f\left(D^{-}\right)$and $f\left(D^{+}\right)$cover the deleted disk $D-v$.

Proof. We let $C^{-}\left(f, e^{i \alpha}\right)$ and $C^{+}\left(f, e^{i \alpha}\right)$ be the lower and upper cluster set with respect to $D^{-}$and $D^{+}$, then both of them are closed sets. There are two cases to be considered: Either $C^{-}\left(f, e^{i \alpha}\right)=C^{+}\left(f, e^{i \alpha}\right)=\bar{D}_{w}$ or not, where $\bar{D}_{w}=\{w:|w| \leqslant 1\}$. We shall begin to settle the first case. To prove the assertion, we suppose on the contrary that there is a value $w \in D_{w}$ for which $w \neq v$ and $w \notin f\left(D^{-}\right)$. We then consider the function $g(z)=1 /(f(z)-w)$. Choose a number $\beta<\alpha$ for which the radial limit $f\left(e^{i \beta}\right) \neq w$, and denote by $R_{\alpha}$ and $R_{\beta}$ the radial segments on which the function $g(z)$ is bounded. Join $R_{\alpha}$ and $R_{\beta}$ by a line segment $L$ such that $g(z)$ is also bounded on $L$. Let $\Delta(\beta, \alpha)$ be the region bounded by $R_{\alpha}, L, R_{\beta}$, and the arc $A(\beta, \alpha)=\left\{e^{i \theta}: \beta<\theta<\alpha\right\}$. Since $w \in C^{-}\left(f, e^{i \alpha}\right)$, it follows that the function $g(z)$ is unbounded in $\Delta(\beta, \alpha)$. By the same argument as Theorem 5, we conclude that the function $f(z)$ has the asymptotic value $w$ either at the point $e^{i \alpha}$ or at a point $P$ arbitrarily close to $e^{i \alpha}$ from below. Since $f(z)$ is bounded, the first case implies that $w=v$, a contradiction, while the last case violates the isolated singularity of $e^{i \alpha}$. This proves the assertion for $f\left(D^{-}\right)$and the same argument yields the assertion for $f\left(D^{+}\right)$.

It remains to settle the case, say, $C^{-}\left(f, e^{i \alpha}\right) \neq \bar{D}_{w}$. Since any analytic function is an open mapping and the set $C^{-}\left(f, e^{i \alpha}\right)$ is closed, it follows that any point $w$ on the boundary of $C^{-}\left(f, e^{i \alpha}\right)$ is omitted by $f(z)$ in a vicinity of $e^{i \alpha}$ relative to $D^{-}$. By choosing one of such points $w \neq v$ and applying the above argument, we can reach the same contradiction. This completes the proof.

\section{REFERENCES}

1. F. Bagemihl and W. Seidel, Koebe arcs and Fatou points of normal functions, Comment. Math. Helv. 36 (1961), 9-18.

2. E. F. Collingwood and A. J. Lohwater, The theory of cluster sets, Cambridge Univ. Press, London, 1966.

3. J. S. Hwang, On an extremal property of Doob's class, Trans. Amer. Math. Soc. 252 (1979), 393-398.

4. On a problem of Lohwater about the asymptotic behaviour of Nevanlinna's class, Proc. Amer. Math. Soc. 81 (1981), 538-540.

5. O. Lehto and K. I. Virtanen, Boundary behaviour and normal meromorphic functions, Acta Math. 97 (1957), 47-65.

6. A. J. Lohwater, On the Schwartz reflection principle, Michigan Math. J. 2 (1953-1954), 151-156.

7. W. Seidel, On the cluster values of analytic functions, Trans. Amer. Math. Soc. 34 (1932), 1-21.

8. On the distribution of values of bounded analytic functions, Trans. Amer. Math. Soc. 36 (1934), 201-226.

9. M. Tsuji, Potential theory in modern function theory, Maruzen, Tokyo, 1959.

Institute of Mathematics, Academia Sinica, Taipei, Taiwan, Republic of China

Current address: Department of Mathematical Sciences, McMaster University, Hamilton, Ontario, Canada L8S 4K1 